Editorial

\title{
Preface and biographic notes for the special issue on graphics for serious games
}

The use of serious games and applications has emerged as a dominating force in training, education and simulation due to the focus on creating compelling interactive environments at reduced costs by adopting its capacity of engaging younger hi-tech generations in the highly motivated gaming environments and to the opportunities offered by the commodity technologies commonly associated with the entertainment industries. This field is informed by theories, methods, applications and the stateof-the-art in a number of areas based on technological principles and innovation, advances in games design, pedagogic methodologies and the convergence of these fields.

This special issue presents a highlight of this diverse community and demonstrates how computer graphics is contributing and being enhanced by seriousgames.

The four papers chosen for this special issue offer a new contribution to the world of graphics, in terms of new tools or adapting more traditional graphic methods for the use in training applications.

"A Head Movement Propensity Model for Animating Gaze Shifts and Blinks of Virtual Characters" by Peters and Qureshi is an extended version of the second IEEE Conference on Games and Virtual Worlds for Serious Applications (VS-Games 2010) best paper. It outlines how to animate more believable in-game characters, modelling the behaviour of gaze shifts and blinks following the characters' attention, resulting in further user engagement with the virtual character, thus affecting learning outcomes and immersion in educational environments.

The Infinitex system by Hollemeersch et al., adopts the concepts of virtual memory for the use in texture memory.

This significantly enhances asset creation in games by giving artists the ability to design arbitrary large textures, increasing productivity and reducing asset re-use, effectively making the gaming experience more immersive. The authors also discuss how to make this work practically within a gaming project.

The two articles on RIST and VFire adopt modern graphics methods used in games to develop systems suitable for training in environments that are considered dangerous. The "RIST: Radiological Immersive Survey Training for Two Simultaneous Users" paper, by Koepnick et al., describes a system for training National Guard Civil Support Teams to respond when dealing with dangerous radioactive materials. "VFire: Immersive Wildfire Simulation and Visualization", by Hoang et al., provides a system for training and educating firefighters and policy makers to combat wild fires, simulating the spread based on real-world data and allowing realistic visualization of the virtual scenarios; both simulation and visualization tasks are computed on a modernGPU.

We hope that this special issue will contribute to foster wider interest in this exciting and emerging field, leading to the emergence of new application areas and encouraging more graphics researchers and practitioners to participate in future original contributions.

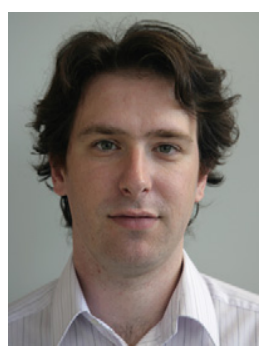

Kurt Debattista is an Assistant Professor at the University of Warwick. He has a Ph.D. in Computer Science from the University of Bristol and an MSc (distinction) by research in parallel computing and systems programming from the University of Malta. He has a first degree in Mathematics and Computing from the University of Malta. His research, published in over 50 papers in journals and international conferences, has focused on high-fidelity rendering, parallel computing, high-dynamic range imaging and seriousgames.

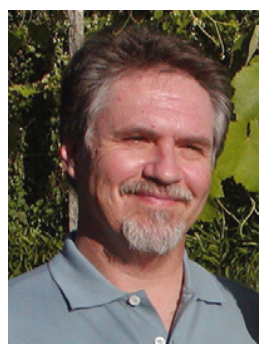

Alberto José Proença is a Professor at the Dep. Informatics, Universidade do Minho, where he has been lecturing since the late 1970s. His main interests since the Ph.D. at UMIST (UK) have been in parallel and scalar computing, with a later focus on heterogeneous environments and specific processing devices, exploring advanced computing features in case studies related to virtual environments (e.g., archaeology) and civil protection (namely forest fires). He headed the University Computer Centre for 15 years and he currently leads the Advanced Computing focus area in the National Cooperation Program between Portugal and the University of Texas in Austin, in close cooperation with the Digital Media field on the same program. He has recently chaired the organization of international conferences in advanced computing and digital media, including VAST'2008 (focus on Virtual Archaeology), VS-Games'2010 (focus on Serious Games) and IBERGRID'2010 (focus on e-Science).

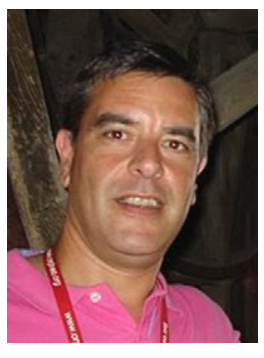

Luís Paulo Santos is an Auxiliar Professor at the Department of Informatics, Universidade do Minho, Portugal. His interests are in interactive high fidelity rendering and parallel processing. He received his Ph.D. in 2001 from Universidade do Minho in Scheduling under Conditions of Uncertainty. Luís Paulo published several papers on both computer graphics and parallel processing on international conferences and journals. He has been a member of several international program committees, acted as program co-chair of the 2007 EGPGV symposium and EuroPar 2005 conference and organized EGPGV 2006 and VS-Games 2010 in Braga, Portugal. He manages several 
nationally funded graphics $R \& D$ projects and participates in several European projects in both academia and industry. He is a member of the Direction Board of the Portuguese Chapter of Eurographics since 2008.

Guest editors

Kurt Debattista*

E-mail address: K.Debattista@warwick.ac.uk
Alberto José Proença E-mail address: aproenca@di.uminho.pt

Luís Paulo Santos E-mail address: psantos@di.uminho.pt

* Corresponding author. 